\title{
Staat und Raum: Theoretische Debatten in der Politischen Geographie
}

\author{
M. Müller \\ Geographisches Institut, Universität Zürich, Zürich, Switzerland \\ Correspondence to: M. Müller (martin@martin-muller.net)
}

Belina, B. (Ed.): Staat und Raum (= Staatsdiskurse, Band 26), Stuttgart, Steiner, 188 pp., ISBN-13: 978-3-515-103466, €42.00, 2013.

Kein Zweifel: Der deutschsprachigen Politischen Geographie geht es gut. Noch in den 1990er-Jahren war die Teildisziplin selbst von Geograph_innen kaum beachtet und oft auf die räumliche Konfliktforschung auf regionaler Ebene reduziert. Doch derzeit erfreut sie sich grossen Zuspruchs. Politische Geographin zu sein ist en vogue - auch unter denen, die sich ansonsten Stadtgeographinnen, Kulturgeographinnen oder Wirtschaftsgeographinnen nennen. 2000 erschien Paul Reubers Bestandsaufnahme neuer Strömungen in der Politischen Geographie, vor allem im anglophonen Raum, in der Geographischen Zeitschrift (Reuber, 2000); 2001 der erste Sammelband, der neue theoretische Ansätze dem Rest der Disziplin nahebrachte (Reuber and Wolkersdorfer, 2001). 2012 erfolgte ein zentraler Anstoss zur Bildung eines neuen Kanons der deutschsprachigen Politischen Geographie mit der Veröffentlichung des Lehrbuchs Politische Geographie (Reuber, 2012; siehe die Besprechung in der Geographica Helvetica in Korf, 2013). Es gibt Sommerschulen, regelmässige Arbeitskreistreffen, eine inzwischen mehr als zehn Bände umfassende Buchreihe - und nun also der nächste Schritt, dieses Mal über die Disziplingrenzen hinweg.

„Staat und Raum“ erschien als Band 26 der Reihe „Staatsdiskurse“, einer ansonsten politikwissenschaftlich ausgerichteten Serie. Thomas Hobbes' Leviathan grüsst mit Schwert und Zepter als personalisierte Staatsgewalt vom Umschlag. Die elf Autor_innen der neun Kapitel präsentieren überwiegend neue theoretische Ansätze der Politischen Geographie im deutschsprachigen Raum - vom Postkolonialismus bis zu Deleuze und Guattaris Gefügen - und ihre Relevanz für die Erfassung des Verhältnisses von Staat und Raum. Die Beiträge arbeiten sich jeweils an einem theoretischen Thema ab, illustrieren die Argumentführung aber auch an einigen Beispielen. Dabei muss man der in anderem Zusammen- hang gemachten Beobachtung Benedikt Korfs (2013) zustimmen, dass die Mehrzahl der vorgestellten Ansätze stark von der anglophonen Debatte inspiriert ist. Diese Inspiration von anderswo mag auch dem fast vollständigen Erliegen generisch deutschsprachiger Theoriebildung und Forschung in der Politischen Geographie nach dem Zweiten Weltkrieg zuzuschreiben sein. Dieser historische Hintergrund und die Rolle der Politischen Geographie als apologetische Pseudowissenschaft zur Legitimation von Machtinteressen und Expansionsbestrebungen werden auch in einem Beitrag (HansDietrich Schultz) thematisiert. Er bildet eine unverzichtbare Folie für den Band, um die speziellen Voraussetzungen und die Grenzen neuerer Theoriebildung zu verstehen.

Mehrere Beiträge im Band kreisen in der ein oder anderen Form um Raumkonstruktion durch Sprache. Sie knüpfen damit explizit oder implizit an den „cultural turn“ in der Humangeographie an, der die Konstruktion von Bedeutung ins Zentrum des Erkenntnisinteresses rückt und im deutschsprachigen Raum mit der Neuen Kulturgeographien Einzug hielt. Antje Schlottmann legt in ihrem Kapitel den Schwerpunkt auf Sprachgebrauch und Sprechakte als performative Praxis. Sprache bildet somit nicht Räume und Grenzen ab; sie erschafft sie. Die Autorin knüpft mit dieser Annahme theoretisch an die Sozialgeographie alltäglicher Regionalisierungen an. Anders als in den meisten Ansätzen der Diskurstheorie wird dabei das Individuum als intentional handelndes und dadurch raumkonstituierendes Subjekt begriffen. Diese Raumkonstitution trennt in der Regel ein vertrautes, nahes Innen von einem bedrohlichen, fernen Aussen. Diese Dichotomie, so argumentiert Schlottmann, sei konstitutiv für das Sprechen von Raum. Auch im Beitrag zur kritischen Geopolitik (Paul Reuber) geht es um binäre Raumkonstruktion durch Sprache, allerdings auf internationaler Ebene. Die kritische Geopolitik versucht offenzulegen wie durch Sprache und Bilder raumbezogene Ordnungsvorstellungen (re-)produziert werden, die politisch kontingentes Handeln naturalisieren und als unvermeidlich darstellen. Der Ansatz 
hat im anglophonen Raum wesentlich zur theoretischen Erneuerung der Politischen Geographie beigetragen. Dabei bemängelt Reuber jedoch den undifferenzierten Akteursbegriff und die begriffliche Unschärfe dieses Ansatzes. Hier mag die Diskurstheorie von Laclau und Mouffe (Iris Dzudzek et al.) hilfreich sein, liefert sie doch ein nuanciertes theoretisches Instrumentarium, um die Herausbildung von Hegemonien durch sprachliche Bedeutungsfixierung zu analysieren. Dabei wendet sie sich jedoch von intentional handelnden Akteuren ab und sieht Subjekte als durch diskursive Strukturen hervorgebracht.

Julia Lossaus Beitrag zu postkolonialen Theorie bildet in mehrerlei Hinsicht eine konzeptuelle Brücke von sprachorientierten zu stärker körper- und materialitätsorientierten Ansätzen im Band. Sie betont die zentrale Stellung von Identität und Differenz in der postkolonialen Theoriebildung und schliesst damit an strukturalistische Ansätze an. Dabei thematisiert sie auch das Bemühen binäre Denkweisen zu überwinden, unter anderem mit Hilfe zahlreicher räumlicher Metaphern wie der des „Zwischenraums“ von Homi Bhabha. Neben dieser Ebene der Bedeutungszuschreibungen arbeitet sie jedoch auch eine praktisch-performative Ebene heraus, bei der die ,körperbezogenen Erfahrungen und habituellen Praktiken“ (S. 103), mit denen Subjekte Geographien und damit Machtungleichheiten produzieren und reproduzieren, im Vordergrund stehen.

Vier weitere Kapitel gruppieren sich, mit unterschiedlicher Schwerpunktsetzung, um theoretische Zugriffe, die Körperlichkeit, Materialität und gelebte Praxis als zentrale Phänomene betrachten. Sie widmen sich damit vorwiegend neueren theoretischen Entwicklungen, die vor allem in der anglophonen Geographie an Bedeutung gewonnen haben. Anke Strüvers Kapitel zu feministischen Ansätzen in der Politischen Geographie konstatiert zunächst ein Paradoxon: Die feministische Geographie nach Ansätzen zur Konzeptualisierung von Staat zu befragen verwundere zunächst etwas, zeichne sich doch diese heterogene Strömung am ehesten durch eine Abkehr von Staat als primärem Untersuchungsgegenstand einer staatszentrierten Politischen Geographie mit grossem „, $\mathrm{P}^{\text {“ }}$ aus. So verweist Strüver auf anti-etatistische Positionen in der feministischen Geographie, die den Staat primär als patriarchales Herrschaftsinstrument sehen, das traditionelle Geschlechterrollen reproduziert. Männern bleibt dabei der öffentliche Raum sowie der Staat, die res publica, vorbehalten, während Frauen auf den privaten Raum und die häusliche Sphäre reduziert werden. Indem die feministische Geographie das Private zum Politikum macht, erweitert sie jedoch den Politikbegriff entscheidend: Dabei rückt im Rahmen einer Reskalierung die Frage der Verschränkung von staatlichem Handeln und der vormals als privat betrachteten Räume in den Vordergrund. Daraus ergibt sich gleichzeitig auch die Forderung nach einer stärkeren Berücksichtigung der Subjektebene in der Forschung, die sich zum Beispiel durch die Fokussierung auf Emotionen als Forschungsgegenstand niederschlägt.
Das Verhältnis zwischen Individuum und Staat steht auch bei Matthew Hannahs Kapitel im Zentrum, in dem er eine hervorragende, prägnante Übersicht über die geographischen Implikationen von Foucaults Machtanalytik gibt. Hannah unterscheidet drei Machtformen in Foucaults Werk - disziplinäre Macht, Biomacht und Gouvernementalität - durch die Territorium und Bevölkerung kalkulierbar und damit regierbar gemacht werden. Am Beispiel von städtischen Visionen und Plänen, dem Einsatz räumlicher Raster sowie territorialer Grenzsicherung diskutiert er den Einsatz und die Verschränkung dieser Machtformen. Von Foucault und seinem Konzept der Dispositive ist es dann ein konsequenter und wichtiger Schritt zu Deleuze und Guattaris Gefügen, die Ulrich Best einführt. Deleuze und Guattaris Werk stellt sicherlich in der deutschsprachigen Politischen Geographie noch viel zu wenig genutzte Ideen zur Verfügung, Materialität und Affektivität zu denken. Gefüge (agencements im französischen Original) sind multiple, sich ständig verändernde Kombinationen aus Mensch und Material, die durch die affektive Kraft von Wünschen (désirs) zusammengehalten werden. „Ihre [Deleuze und Guattaris] ganze Theorie baut darauf auf, zu untersuchen, wie durch ständige Rückkopplung von Wünschen stabilere Gesamteinheiten entstehen, die dann selbst wieder versuchen Wünsche auf sich zu territorialisieren“ (S. 153). Staat kann demnach als ein Gefüge der Gefüge betrachtet werden, das dadurch Stabilität und Wirkmächtigkeit erlangt, dass es (unerfüllbare) Wünsche auf sich vereint. Dass diese Wünsche unerfüllbar bleiben und also stets einen Mangel aufweisen, garantiert die Dauerhaftigkeit affektiver Bindung.

Eine Lanze für die Materialität des Staates bricht schliesslich Bernd Belina, in Abgrenzung zu rein diskurstheoretischen Ansätzen. Mit der Verwendung des Begriffs der Materialität greift er ein derzeit viel diskutiertes Konzept auf, wendet es jedoch geschickt in Richtung einer materialistischen Staatstheorie. Mit Materialität bezeichnet er dabei zum einen primär die durch soziale Praxis produzierte eigenständige Existenz und organisatorische Festigkeit des Staates in Form von Routinisierungen und Institutionalisierungen, zum anderen aber auch die physisch-materielle Dinglichkeit des Staates. Die Konstitution von Staatlichkeit funktioniere demnach mittels materialisierter Apparate auf der Basis von Gewalt (z.B. Militär, Polizei) und Ideologie (z.B. Medien, Schulen). Mit Hilfe dieser Apparate erlange der Staat Territorialität durch Abgrenzung eines Territoriums, beispielsweise in Reaktion auf ökonomische Anforderungen, Sicherheitsbzw. Versicherheitlichungsdiskurse oder kulturelle Hegemonien.

Staat und Raum führt die Leserin in seinen neun Beiträgen an den Stand der theoretischen Debatten um Staat und Staatlichkeit in der Politischen Geographie heran. So ist der Band hervorragend geeignet für all diejenigen, seien es Student_innen oder Fachkolleg_innen, die auf anspruchsvollem Niveau einen Einblick in das 
derzeitige Forschungsgeschehen der deutschsprachigen Politischen Geographie erhalten wollen.

Trotz interdisziplinären Anspruchs der Reihe wirkt der Band stark für Geograph_innen gemacht. Er referiert primär geographische Literatur und Debatten, reflektiert über die eigene Disziplingeschichte - und versäumt es dabei manchmal hinüber in die Politikwissenschaft zu blicken und Anschlussmöglichkeiten auszuloten. Diskurstheorie, Feminismus, Gefüge, Postkolonialismus sind jedoch allesamt Themen, die auch Politikwissenschaftler_innen beschäftigen. Zwar sind sie in jenem Feld nicht, wie in der Geographie, im theoretischen Mainstream, aber gerade deshalb wäre es eine gute Gelegenheit gewesen, auf die Perspektiven wechselseitiger Befruchtung einzugehen, bei der die Geographie zur Abwechslung einmal nicht importieren sondern vielleicht auch exportieren könnte. Aber die Erfolgsgeschichte der Politischen Geographie der vergangenen fünfzehn Jahre gibt uns Grund genug zur Annahme, dass es nicht lange dauern wird, bis es heisst: Fortsetzung folgt!

\section{Literatur}

Korf, B.: Gibt es (noch) eine deutschsprachige Politische Geographie?, Geogr. Helv., 68, 73-75, doi:10.5194/gh-68-73-2013, 2013.

Reuber, P.: Die Politische Geographie als handlungsorientierte und konstruktivistische Teildisziplin: angloamerikanische Theoriekonzepte und aktuelle Forschungsfelder, Geogr. Z., 88, 36-52, 2000.

Reuber, P.: Politische Geographie, UTB, Stuttgart, 2012.

Reuber, P. and Wolkersdorfer, G.: Politische Geographie: handlungsorientierte Ansätze und Critical Geopolitics, in: Heidelberger Geographische Arbeiten, Geographisches Institut der Universität, Heidelberg, 2001. 